\title{
Electron Conductivity on Helium Films
}

\author{
J. Klier • I. Doicescu • P. Leiderer • V. Shikin
}

\begin{abstract}
Electrons on liquid helium film form a two-dimensional (2D) array with a wide range of electron density. This system is also very interesting for applications in restricted geometry. The conductivity $\sigma$ of the electron arrays, however, strongly depends on the thickness $d$ of the helium film adsorbed above solid substrates. This behaviour of $\sigma$ is discussed in detail for a randomly rough substrate. It turns out that for the dependence of the conductivity $\sigma(d)$ there exist three regions of helium thicknesses: $d>d_{\text {min }}, d \sim d_{\text {min }}$, and $d<d_{\text {min }}$. Here $d_{\text {min }}$ is the helium fil thickness which corresponds to a relatively deep minimum of the $2 \mathrm{D}$ conductivity. In the firs interval, $d>d_{\min }$, a two-fraction scenario determines the behaviour of $\sigma(d)$. In the vicinity of $d_{\min }$ percolation phenomena develop and the conductivity exhibits different types of the so-called dip effect. For even thinner helium films i.e., when $d<d_{\text {min }}$, an activation type of mobility is stimulated. The presented model fit quite well to existing data of $a c$ and $d c$ electron mobility.
\end{abstract}

Keywords 2D electron systems - Thin helium film · Electronic transport - Surface roughness

PACS $67.70 .+\mathrm{n} \cdot 72.10 .-\mathrm{d} \cdot 72.60 .+\mathrm{g} \cdot 73.50 .-\mathrm{h}$

\section{Introduction}

Two-dimensional (2D) electron arrays on thin helium film (2DES) are challenging systems since they are sensitive to many different parameters. Such are the dielectric 
constant both of the helium fil and the substrate, the characteristics of their van-derWaals interaction $\Delta C_{3}$, the temperature $T$, the $2 \mathrm{D}$ electron density $n_{s}$, the pressing electric fiel $E_{\perp}$, the level of substrate roughness, and so on. Most of these parameters can be regarded as known ones. However, in the case of the substrate roughness, this characteristic is difficul to determine. As a rule it has to be extracted from experimental data. Some qualitative possibilities of such diagnostics, using the dynamics of the 2DES, are presented below.

\section{Corrugation Features}

We start from the periodically corrugated surfaces. Corrugation is a special type of substrate roughness $\delta(x)$, i.e., the amplitude of perturbation has the limitation from the top: $|\delta(x)|<\delta_{0}$, see Fig. 1. An investigation of this type of surface perturbation reveals two typical phenomena - the 'screening' effect and the electron localizationapplied to the system '2DES + surface roughness of substrate'.

Figures 1 and 2 show that the thickness $\zeta(x)$ of the helium fil does not follow the profil $\delta(x)$ (screening effect). The screening radius $R$ (i.e., the Laplace radius, caused by the finit surface tension) can be large $(R \gg b$; strong screening) or small ( $R<b$; weak screening). $R$ is define as $R=\alpha /(\rho g h)$, where $\alpha$ and $\rho$ are the surface tension and density of the liquid helium film $g$ is the acceleration due to gravity, and $h$ is the distance between the bulk and average plane of the distribution $\delta(x)$. In Fig. 2 the measured shape of the helium meniscus and a fi of the experimental data are shown for the structure from Fig. 1, with $b=300 \mu \mathrm{m}$ and $h=0.42 \mathrm{~mm}$, see [1].

The second effect is the localization of some electron fraction along the thin part of the helium fil (e.g. along distance $a$ in Fig. 1). So the total electron density $n_{s}$ can be presented as the sum

$$
n_{s}=n_{l}+n_{e}
$$

where $n_{l}$ is the trapped electron fraction with low mobility and $n_{e}$ is the free electron fraction along the thick part of the helium film Figure $2 b$ indirectly shows the existence of the two electron fractions: without a trapped electron fraction on the tops of

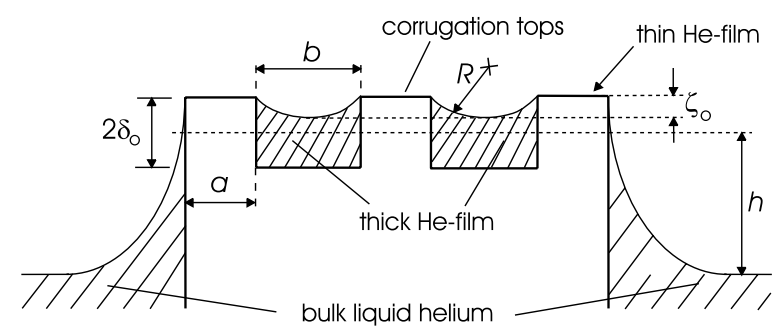

Fig. 1 Schematical sketch of a corrugated surface where, due to capillary condensation, a suspended thick liquid helium fil exists. $\delta(x)$ is the corrugation profile $(a+b)$ is the corrugated structure period, and $\zeta(x)$ is the profil of the helium film $R$ is the radius of curvature of the capillary condensed thick helium fil and $h\left(>2 \delta_{o}\right)$ is the distance between the average structure of the substrate and the bulk liquid helium surface 


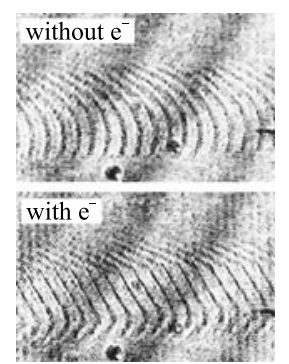

(a)

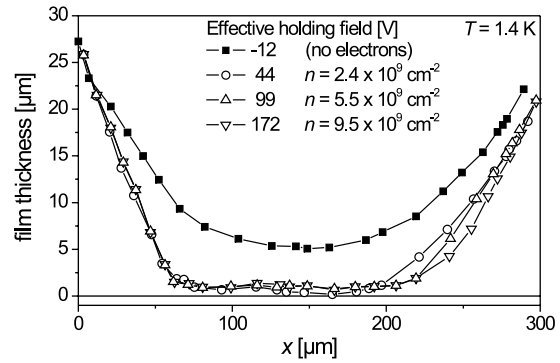

(b)

Fig. 2 (a) Video image of interference fringes resulting from a suspended helium film $h=0.42 \mathrm{~mm}$ and the pressing fiel $E_{\perp}=172 \mathrm{~V}$. Top: uncharged film Bottom: charged with electrons. (b) Film thickness versus position within channel, determined from the video images. Due to the electron pressure the helium fil is thin in the center of the channel. For large electron densities the width of the thin fil region does not increase any more. For details see [1]

Fig. 3 (a) Helium fil profile for different $R(h)$ :

$R_{A}>R_{B}>R_{C}$. (b) Balance of forces holding the electron on the helium surface: $e E_{\perp}$ is the electric force due to non-zero pressing fiel $E_{\perp}$ and $F_{\text {im }}$ is the image force

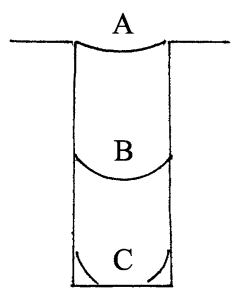

(a)

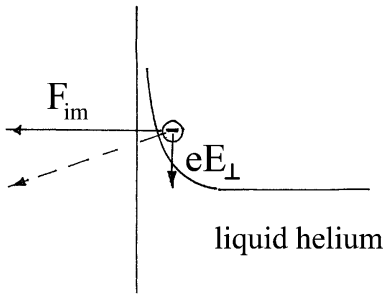

(b)

the channel structure an increase of $n_{s}$ should result in an increase of the width of the electron area at the bottom within the channel. This, however, is not seen in the measurements, see [1].

Qualitatively interesting is the extreme case of relatively low density of 2D electrons (opposite to that presented in Fig. 2) where

$$
n_{s}<a^{2}, b^{2}
$$

i.e., there are less than one electron per corrugated lattice cell. Let us consider a fragment of such a lattice shown in Fig. 3a. It is clear that different values of $R(h)$ correspond to different helium filling of the well changing from completely fille (meniscus A) to practically empty one (meniscus C). The problem is to fin out where the electron resides which happened to occur in the indicated lattice fragment. In the absence of electric fiel $E_{\perp}$ pressing the electrons to the liquid helium surface the most energetically favorable places are located at the peaks of the $\delta(x)$ profil (here the helium fil thickness is minimal). On the contrary, if $E_{\perp} \neq 0$, the electron position is governed by the balance of forces indicated in Fig. 3b. Electron is localized in the transition domain of the helium surface profil $\zeta(x)$ so that the net force is normal to the fil surface. Without going into details one can state, bearing in mind the experiments discussed below, that electrons are indeed localized following the mechanism presented in Fig. $3 \mathrm{~b}$ descending into the $\delta(x)$ profil as $R(h)$ is reduced. 


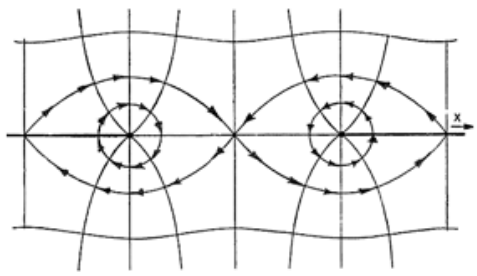

(a)

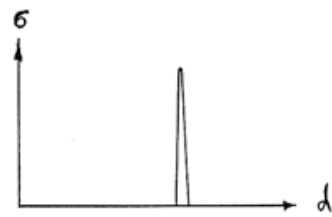

(b)

Fig. 4 (a) Trajectories of 1D electrons on the periodical surfaces of $\delta(x, y)$ with different curvatures and the corresponding separatrix. It is clearly seen that the separatrix is the only trajectory along which electrons can travel from cell to cell. (b) Electron conductivity for the system of 1D electrons as a function of helium fil thickness $d$

The arising picture results in interesting geometry of localized states on the twodimensional lattice $\delta(x, y)$. Its fragment along one of the directions is shown in Fig. 4a. Obviously, in the vicinity of maxima and minima of $\delta(x, y)$ the trajectories of 1D electrons are closed and span the peaks of $\delta(x, y)$ (clockwise arrows) or prove to be "inserted" into the minima of $\delta(x, y)$ (anticlockwise arrows). The only exception is a single plane containing the separatrix separating the states with different curvatures. Moving along the separatrix, the 1D electrons can travel between the cells thus producing a finit conductivity along the corrugated helium film Qualitatively, the resulting conductivity $\sigma$ as a function of the average thickness of the helium fil in the well demonstrates the behaviour depicted in Fig. 4b. We believe that this property obviously taking place for the periodic corrugation should also be valid for the substrates with arbitrary roughness.

\section{Random Substrate Roughness}

As a rule the roughness cannot be presented via simple corrugation. The suitable alternative is the introduction of Gaussian roughness. Under these conditions $a c$ and $d c$ conductivities inevitably start to be sensitive to the average helium fil thickness $\langle d\rangle$ due to the substrate roughness. To explain the situation we need the basic Gaussian roughness definitions Mainly the one-dimensional presentation is sufficient However, some qualitative statements are valid just in the $2 \mathrm{D}$ roughness picture.

We now consider a random one-dimensional roughness $\delta(x)$ with a Gaussian distribution of amplitudes $D(\delta)$ and autocorrelation function for $\delta(x)$, see [2]:

$$
D(\delta)=\frac{\exp \left(-\frac{\delta^{2}}{2 \Delta^{2}}\right)}{\left(2 \pi \Delta^{2}\right)^{1 / 2}} \quad \text { and } \quad\left\langle\delta(x) \delta\left(x-x^{\prime}\right)\right\rangle=\Delta^{2} \exp \left(-\frac{x^{\prime 2}}{2 \eta^{2}}\right)
$$

where $\Delta^{2}=\left\langle\delta^{2}\right\rangle$ is the mean-square deviation of the roughness amplitude and $\eta$ is the correlation length.

Using (2) one obtains

$$
\left\langle\delta^{\prime \prime} \delta^{\prime \prime}\right\rangle=\beta^{2} \equiv \frac{3 \Delta^{2}}{\eta^{4}} \quad \text { and } \quad D(\gamma)=\frac{\exp \left(-\frac{\gamma^{2}}{2 \beta^{2}}\right)}{\left(2 \pi \beta^{2}\right)^{1 / 2}}, \quad \gamma=\delta^{\prime \prime} .
$$


The definition in (3) are used below for introducing the distribution of the continuum electron oscillator frequencies $\omega_{r}$, caused by interaction between electrons and a rough solid substrate: $\omega_{r}^{2} \propto \delta^{\prime \prime}$.

Besides we need the correlation function [2]

$$
P\left(\delta, \delta^{\prime \prime}\right)=\left\langle\delta \delta^{\prime \prime}\right\rangle=\frac{\eta^{2}}{2^{3 / 2} \pi \Delta^{2}} \exp \left(-\frac{\delta^{2}}{2 \Delta^{2}}\right) \exp \left[\frac{\left(-\delta+\delta^{\prime \prime} \eta^{2}\right)^{2}}{4 \eta^{2}}\right]
$$

which is necessary in the discussion of the activation type mobility.

In the presence of roughness, (2), there are three essential intervals of the helium fil thickness:

(a) $\kappa^{-1} \geq R \geq \eta$; this interval is suitable for the two-fraction separation in the $2 \mathrm{D}$ electron system, $\kappa^{2}=\rho g / \alpha$;

(b) $R \simeq \eta$; in this situation the development of an infinit conducting cluster becomes possible (so-called dip effect);

(c) $R \ll \eta$; the area for the activation type conductivity.

Here $R$ is the Laplace radius, with

$$
\frac{2 \alpha}{R} \simeq \rho g^{*} h,
$$

where the height $h$ (distance between substrate and bulk liquid level) is define in Fig. 1, and $g^{*}$ is the effective gravity acceleration with $g^{*}=g+\Delta C_{3} / \rho d^{4}$.

All these limiting cases are interesting and have to be discussed separately.

\subsection{Two-Fraction Situation}

If the helium fil is thick then just a few roughness tops will be at the level (or above) of the free helium surface. The $2 \mathrm{D}$ density of these so-called active tops $n_{a}$ is [2]

$$
n_{a}^{-1} \simeq b_{a}^{2}, \quad R \simeq \frac{b_{a}}{2 \sqrt{2}}\left(\frac{b_{a}}{\Delta}\right) \sqrt{\ln \left(\frac{b_{a}}{s \eta}\right)}
$$

with $s^{2}=2 \pi, b_{a}^{2}>\Delta^{2}$, and $R \gg \Delta$ is define in (5), $\eta$ is the correlation length from (2).

Definitio (6) helps to introduce the two-fraction scenario for the electron behaviour. As in (1) we have $n_{s}=n_{l}+n_{e}$, where

$$
n_{l}=\frac{n_{a}}{\exp \left(\frac{V_{a}-\mu_{0}}{T}\right)+1}, \quad V_{a} \geq-\frac{\Lambda}{d_{a}}, \quad \Lambda=\frac{e^{2}\left(\epsilon_{d}-1\right)}{4\left(\epsilon_{d}+1\right)}, \quad d_{a}^{3} \simeq \frac{\Delta C_{3}}{\rho g h}
$$

and

$$
n_{e}=\frac{n_{e}^{0} \exp \left(\frac{T_{e}}{T}\right)}{\exp \left(-\frac{\mu_{0}}{T}\right)+1}, \quad n_{e}^{0}=\frac{m T}{2 \pi \hbar^{2}}, \quad T_{e}=\frac{e E_{\perp} \Delta}{2 \sqrt{2}\left[\ln \frac{(R \Delta)^{1 / 2}}{\eta}\right]^{1 / 2}},
$$




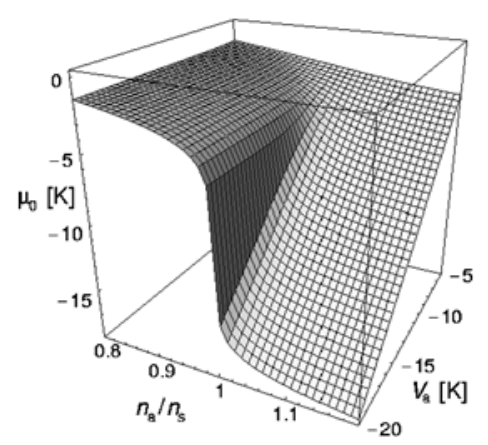

(a)

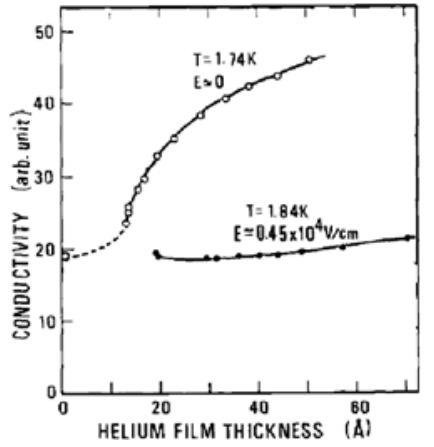

(b)

Fig. 5 (a) The electro-chemical potential, $\mu_{0}$, as function of coupling energy, $V_{a}$, and density ratio of the number of localized electrons due to the active tops, $n_{a}$, to the total number of electrons, $n_{s}$. When $n_{a}=n_{s}$ then there is a sharp drop in $\mu_{0}$, which increases as the coupling energy gets bigger, see [3]. (b) Electron conductivity against the helium fil thickness for two pressing electric fields Reproduced from [4]. In contrast to Figs. 8 and 9, these data do not show the dip effect

where $\Delta C_{3}$ is the constant of van-der-Waals interaction. The fraction $n_{e}$ corresponds to the free electron motion along the helium surface. In Fig. 1 this fraction is distributed along the minimum of the meniscus. The second fraction, $n_{l}$, represents the density of electrons localized to potential wells of the roughness of the solid substrate. These electrons are localized in the vicinity of the tops with density $n_{a}$ above the helium film The total density $n_{s}$ is usually fi ed. The ratio between $n_{e}$ and $n_{l}$ is controlled by the potential $V_{a}$, temperature $T$, and the electro-chemical potential $\mu_{0}$. The dependence of $\mu_{0}$ is shown in Fig. 5a, see [3].

In terms of $n_{e}$ and electron mobility $\mu$ the $d c$ conductivity $\sigma$ can be written as

$$
\sigma=n_{e} \mu .
$$

Definition (8) and (9) show, at least qualitatively, that $\sigma$ decreases monotonically when the thickness of the helium fil gets thinner. Indeed, the mobility $\mu$ goes down with decreasing thickness of the helium fil and the fraction $n_{e}$ gets smaller. Experiments demonstrate the expected tendency in $\sigma(d)$, see Fig. 5b and [4]. However, from the monotonic behaviour of $\sigma(d)$ it is difficul to deduce the contribution due to $\mu(d)$ and $n_{e}(d)$.

A more informative example of the two-fraction scenario is given by cyclotron resonance (CR) measurements on thin helium films see Figs. 6 and 7. In the twofraction area the trapped electrons cannot participate in the $d c$ conductivity (see (9)). However, these electrons contribute to the $a c$ dynamics seen in the eigenfrequencies $\omega_{r}^{2} \sim \delta^{\prime \prime}$. Therefore the effective absorption CR line has two contributions: one from the free electron motion, and one from the trapped electron states. For a Gaussian distribution of the random potential the trapped fraction continuously grows as $d$ gets thinner. Consequently, the fraction $n_{e}$ shows the opposite behaviour. The experimental confirmatio of this prediction is demonstrated in Figs. 6 and 7 [5].

The data in Fig. 6 show the two contributions to the electron density: the free electron part which decreases with decreasing helium fil thickness and the trapped 


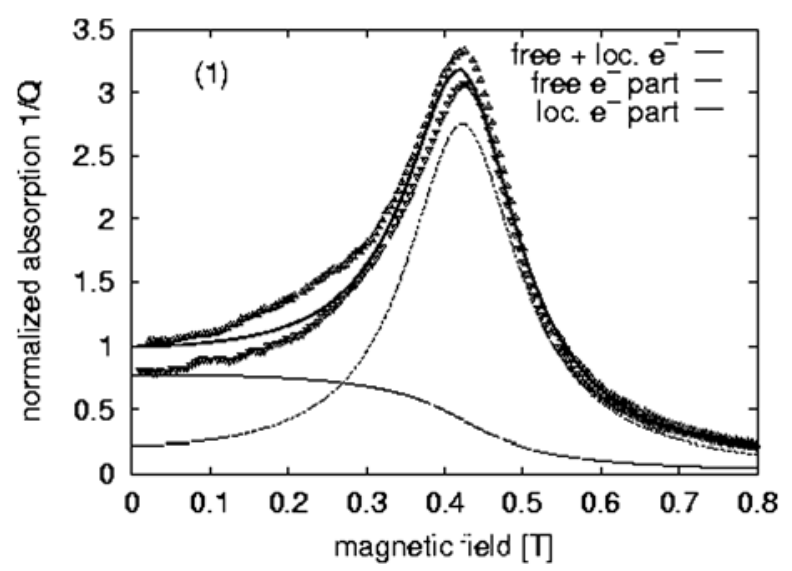

(a)

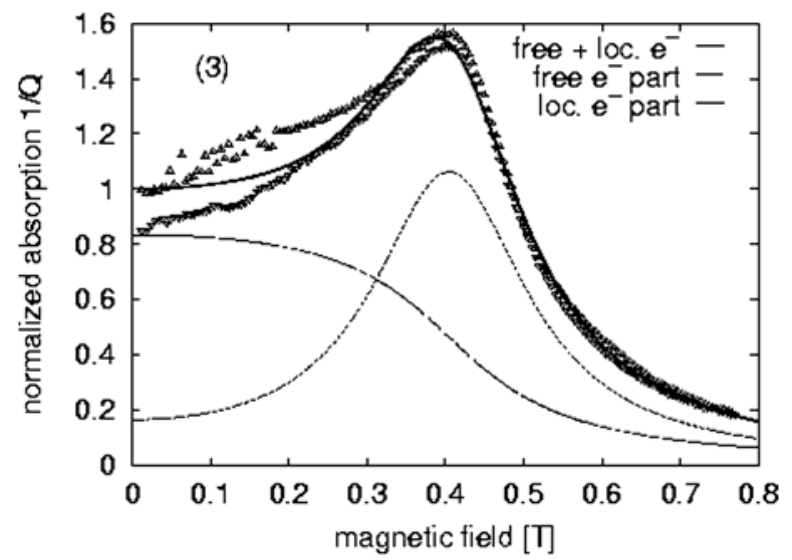

(b)

Fig. 6 The absorption $Q^{-1}$ as function of magnetic fiel for up $(\triangle)$ and down $(\nabla)$ sweeps. The dashed and dotted lines represent the free and localized electron fraction, the full line is the sum of both fitte to the data. From (a) to (b) the helium fil thickness decreases. In (a) $n_{e} \approx 67 \%$ and in (b) $n_{e} \approx 49 \%$. For details see [5]

electron part with opposite tendency. To prove this interpretation it is necessary to express all components of the absorption kinetics in terms which are sensitive to Gaussian roughness. Using the motion equation in a Drude form with the parameter $\tau$, the statistical information (2), (3), and (5) to (8) with the parameters $\Delta$ and $\eta$, and the excitation scenario neglecting the resonator features, one can fi the data using $\Delta=8 \mathrm{~nm}, \eta=6 \mathrm{~nm}, n_{s}=10^{9} \mathrm{~cm}^{-2}$, and $\omega \tau \approx 4$, see [5].

\subsection{Percolation Area, Dip Effect in $d c$ Mobility}

Now let us turn to the reproducibly observable N-shaped feature in the $\sigma(d)$ plot for electrons above the helium fil on rough substrates (the so-called dip effect). This remarkable effect firs observed by Andrey [6] for electrons above the helium 
Fig. 7 Dependence of free electron fraction $n_{e}$ as function of $h$. (•) and (o) are from fittin to all measured data. Both in (a) and (b) the solid lines present the best fi with the same parameters of $a$ and $T_{o}$. The dashed and dotted lines in (a) show fit with different $T_{o}$ but keeping $a$ fi ed, and in (b) with fi ed $T_{o}$ but varying $a$. This shows the good agreement with one set of parameters to describe the measured data. The (o), labeled (1) to (3), correspond to the same data-points as shown in Fig. 6. For details see [5]

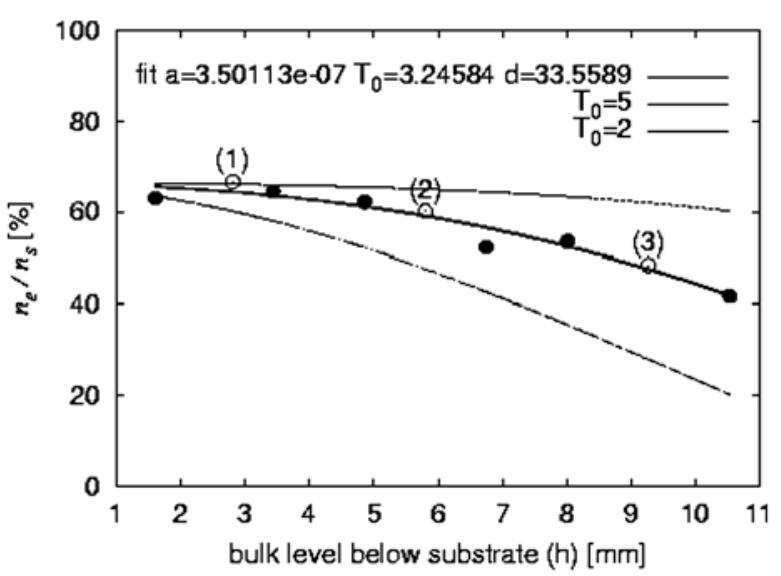

(a)

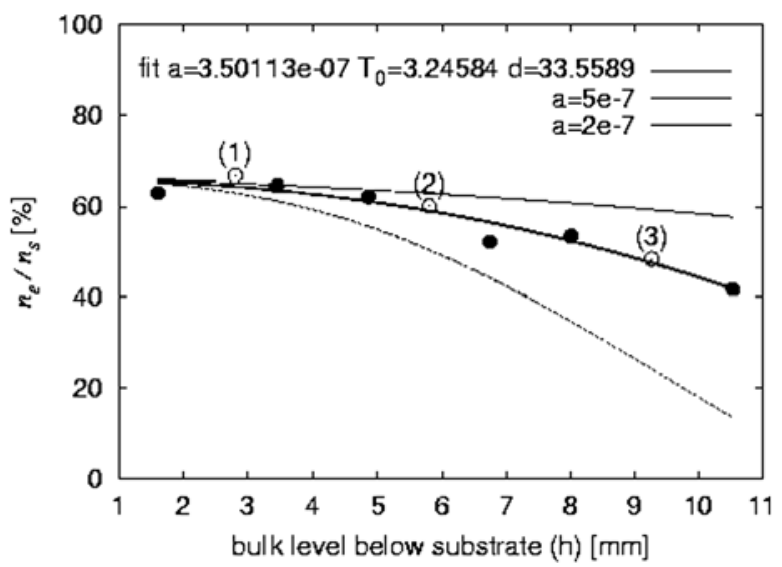

(b)

fil on the sapphire substrate is clearly seen on Fig. 8b. Further independent results presented in Fig. 8a [7] demonstrate the importance of roughness (the dip effect is reduced as the substrate made of solid hydrogen employed in [7] is annealed, i.e. the amplitude of $\delta(x, y)$ is decreased). One should also note a series of measurements of the $d c$ conductivity in the cell firs providing this possibility for electrons above helium (see [3, 8] and Fig. 9). Most important here are the data presented in Fig. 9a revealing a gap between the threshold of the 2D conductivity from the side of $d>$ $d_{\min }$ and its peak in the region of $d<d_{\min }$ indicating the composite nature of the dip effect (here $d_{\min }$ is the threshold thickness for the appearance of 2D conductivity).

Our CR data on the two-component behaviour of 2D electrons on the fil above a rough substrate plotted in Figs. 6 and 7 allow to explain a sharp fall of the 2D conductivity in the domain of

$$
n_{a}>n_{s} \quad \text { and simultaneously } \quad d<d_{\min }
$$


Fig. 8 (a) Conductivity versus temperature measured for a growing helium fil on a freshly prepared quench-condensed hydrogen $\mathrm{f} 1 \mathrm{~m}$ (lower curve) and after thermal annealing of the hydrogen $\mathrm{flm}$ (upper curve). Reproduced from [7].

(b) Mobility as function of helium fil thickness on sapphire as substrate at two different electron densities. Reproduced from [6]

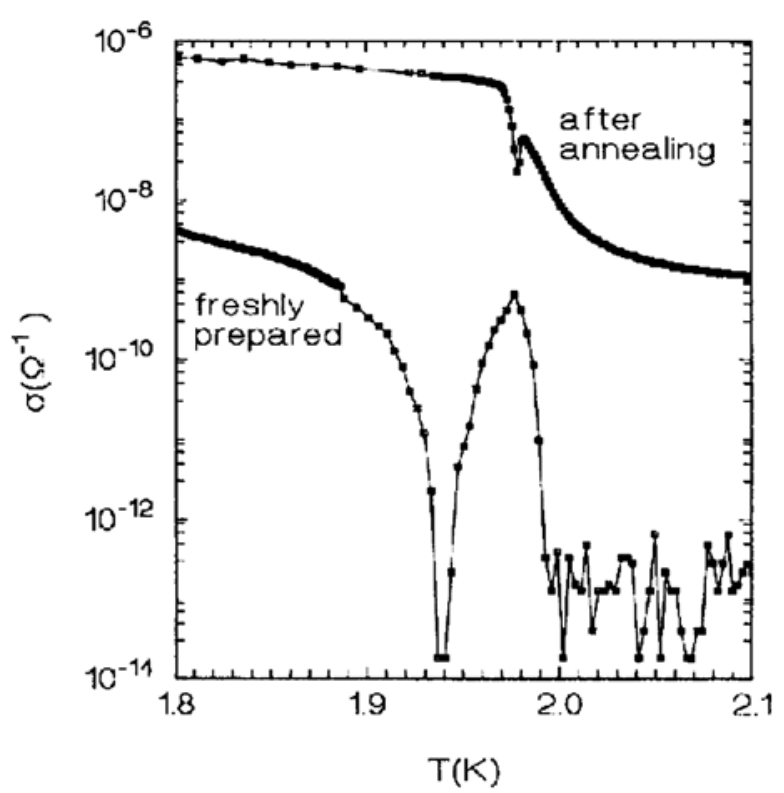

(a)

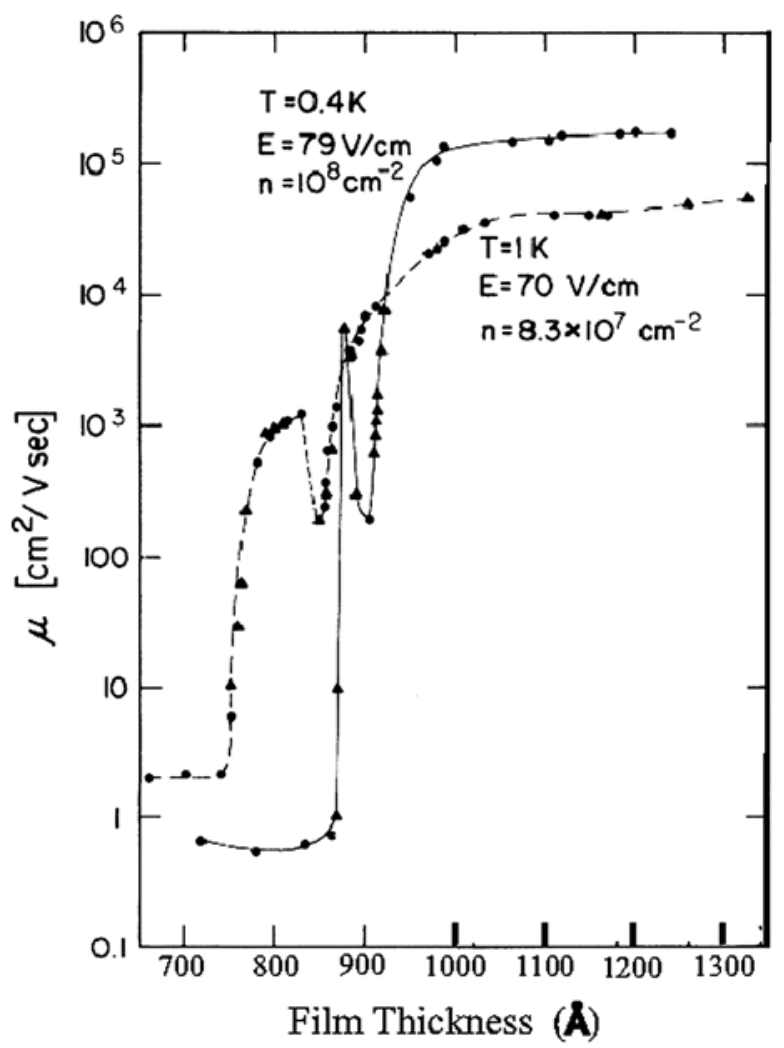

(b) 
Fig. 9 Measurement of $d c$ currents of electrons travelling along a helium film adsorbed on different substrates. The currents are proportional to the $d c$ conductivity. (a) The data show the existence of a quasi-zero conductivity for a finit interval of helium thickness [8]. (b) Dip in conductivity measured on helium film adsorbed on a $\mathrm{Au}$, $\mathrm{Cu}$, and, as shown in the inset, on a Si substrate. For details see [3]

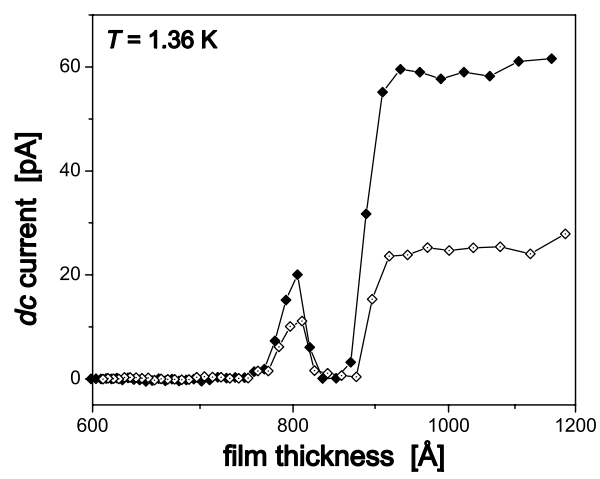

(a)

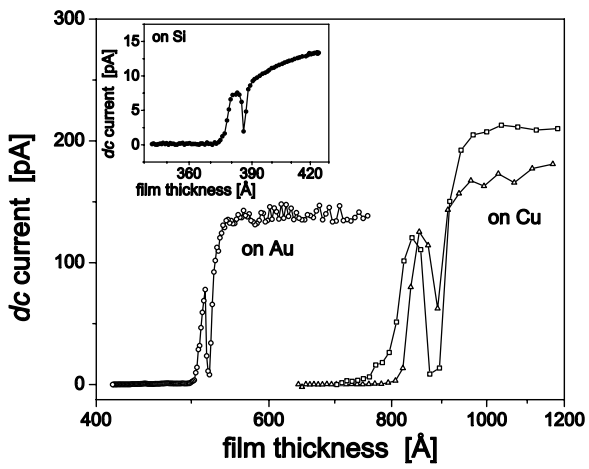

(b)

(for the chemical potential shown in Fig. 5a the fraction $n_{e}$ of free electrons in this domain becomes exponentially small).

However, what is the origin of the sharp rise in $\sigma(d)$ in the range of $d<d_{\text {min }}$ ? We believe that just as for the periodic corrugation shown in Fig. 4a, rough substrates also possess the $\delta$-percolation effect (similar to that shown in Fig. 4b) for 1D localized electrons in the domain of $d<d_{\text {min }}$. We do not attempt to prove the existence of $\delta$-percolation effect in the conductivity of $1 \mathrm{D}$ electrons above the helium fil on an arbitrary rough substrate. Even the ordinary percolation involving the proof of the existence of the threshold is a tough mathematical problem which was only rigorously analyzed with numerical methods [9]. However, by adopting this hypothesis one obtains a natural explanation of the dip effect through a simple combination of two percolation phenomena. One of them is the 2D percolation with the threshold (10), and the second one the 1D percolation with the peak in $\sigma(d)$ corresponding to the helium level crossing the averaged saddle point of the $\delta(x, y)$ profile Schematic picture of this combination of the two contributions to the conductivity is presented in Fig. 10. The thresholds $d_{\min }$ and $d_{\text {peak }}$ are generally different. For $d_{\text {min }}>d_{\text {peak }}$ we have the situation of Fig. 9a. If $d_{\min } \geq d_{\text {peak }}$ then Figs. 8 and $9 \mathrm{~b}$ are realized. Finally, if $d_{\text {min }}<d_{\text {peak }}$, the dip effect may be absent (see Fig. $5 \mathrm{~b}$ ). 


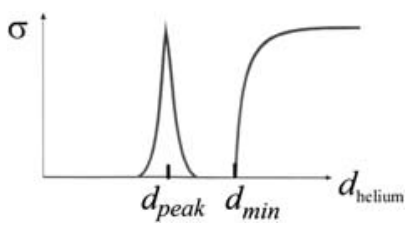

Fig. 10 Schematic structure of the dip effect in the $\sigma(d)$ behaviour. In the range of $d>d_{\min }$, the two-fraction $d c$ conductivity is developed. For $d<d_{\text {min }}$, the 1D percolation around $d=d_{\text {peak }}$ may occur

Fig. 11 Logarithm of the 2D electron mobility on thin liquid helium film with different substrates as a function of $d^{-2}$ Reproduced from [11] (and see references therein)

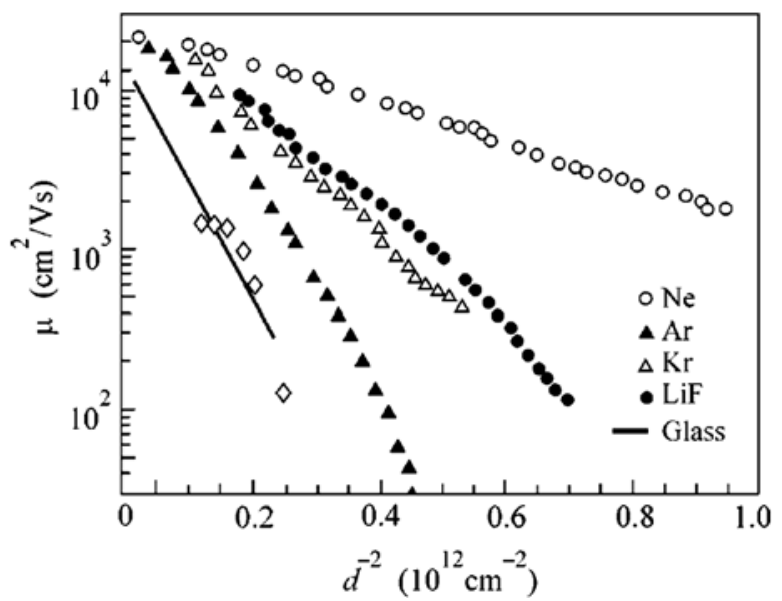

\subsection{Activation Type of Mobility}

For the limit $R \ll \eta$ all electrons are practically trapped below the $1 \mathrm{D}$ percolation level (see Fig. 3a, situation "C") in the minimum of $\delta(x)$. In this case the optimal minimum $\delta_{*}$ follows from (4) and the correct description of the curvature of this effective minimum. We believe that $\delta_{*}^{\prime \prime}$ coincides with the inverse screening radius $R_{*}^{2} \simeq \alpha / \rho g^{*}$. If so, the value $\delta_{*}$ follows from

$$
\frac{\partial P\left(\delta, \delta_{*}^{\prime \prime}\right)}{\partial \delta}=0
$$

where $P(x, y)$ is from (4). After calculations we have from (11) the condition $\delta_{*}=$ $-2 \eta^{2} /\left(3 R_{*}\right)$.

The activation energy is $E_{a} \simeq e E_{\perp}\left|\delta_{*}\right| \propto d^{-2}$ [10]. This result explains the experimental data in Fig. 11 and supports the general picture of the contribution of surface roughness in the conductivity $\sigma(d)$.

\section{Conclusions}

We have shown that surface roughness of the substrate supporting a helium fil can strongly influenc the conductivity of a 2DES on top of the film The model which 


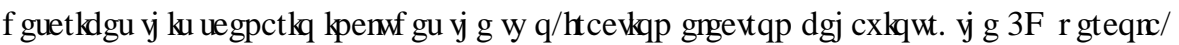
WRQSKHQRP HQRQ DOG DQDFWDWRQWSHRI P REICWW 7 KHVWIIUFWRQ VFQDUR FDQ

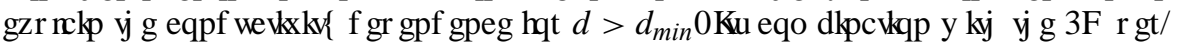
FRDWRQ KHSVUR H S(DQ WHH LMQFHRI WXHS HIFW) RU $d<d_{\text {min }}$ WHP REICWW

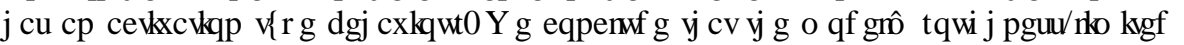

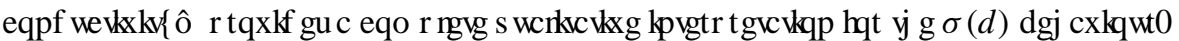

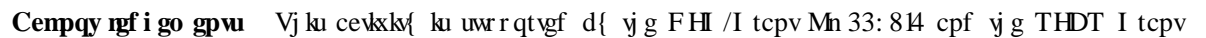

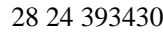

\section{HHHQFN}

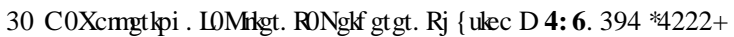

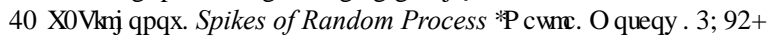

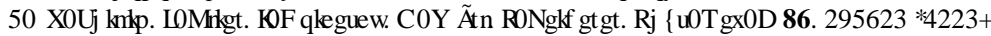

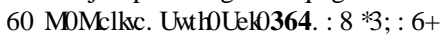

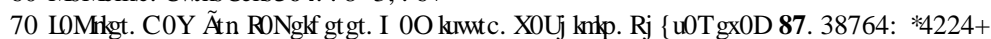

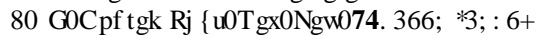

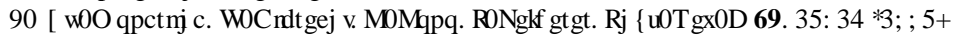

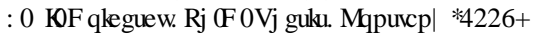

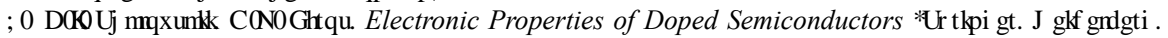
प्य००

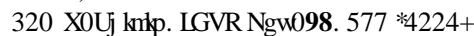

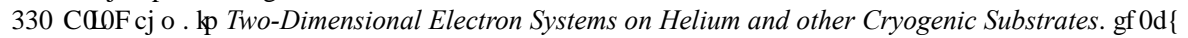

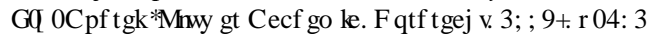

